\title{
Sequentiality and Non-Tensed Verbal Coordination in Korean*
}

\author{
SAE-YOUN CHO \\ Honam University
}

\section{Introduction}

Lakoff (1986) argues that the Across-The-Board (ATB) constraint on extraction from coordinate structures of Ross (1967) is not a syntactic constraint but a semantic or pragmatic one, since ATB violations occur when conjuncts are interpreted sequentially, as shown by the contrast between (1a) and (1b).

a. Which whisky ${ }_{i}$ did Johnny [[go to the store] [and buy i]]?

b. *What ${ }_{i}$ does Johnny [[like apples] [and hate i ]]?

However, Lakoff's (1986) claim that the sequential reading alone suffices to sanction ATB violations cannot be supported in Korean verbal coordination of Tensed Phrases (TPs), where all conjuncts contain tense inflection. As shown in (2), extraction out of the conjunction of TPs in Korean is not possible regardless of whether the conjuncts are interpreted sequentially or non-sequentially.
a. Marcia-ka [imsin-ul ha-ess-ko]
[kyelhoyn-ul hay-ess-ta.]
M-Nom pregnant do-Past-Conj marriage-Acc do-Past-Decl
'Marica was pregnant and got married.' (sequential (SEQ) or non-sequential (NS))
b. *kyelhoyn-ul $i$, [Marcia-ka [imsin-ul ha-ess-ko] $\quad$ [__ ${ }_{i}$ hay-ess-ta.]] marriage-Acc M-Nom pregnant do-Past-Conj do-Past-Decl

Unlike the conjunction of TPs in Korean, ATB violations are allowed in the coordination of Non-Tensed Phrases (NTPs), where the non-final conjuncts lack tense inflection, so that the suffix $-k o$ is directly attached to the verbal root only when the conjuncts are interpreted sequentially, as in (3).

\footnotetext{
* I am grateful to the $30^{\text {th }}$ BLS participants at UC Berkeley, including Alec Marantz, Chung-Hye Han, and Seung-Ho Nam for their discussions and valuable comments, and also to James Yoon, Jong-Bok Kim, and Peter Sells for their critical comments. All misinterpretations and misunderstandings are of course mine. This work was supported by the Korea Research Foundation (Grant Number 2003-041-A00224).
} 

a. Marcia-ka [imsin-ul ha-ko] [kyelhoyn-ul hay-ess-ta.] M-Nom pregnant do-Conj marriage-Acc do-Past-Decl 'Marica was pregnant and got married.' (SEQ or NS)
b. kyelhoyn-ul ${ }_{i}$, [Marcia-ka [imsin-ul ha-ko] marriage-Acc M-Nom pregnant do-Conj $i$ hay-ess-ta.]] 'Marica was pregnant and got married.' (SEQ only) do-Past-Decl

Thus, the facts of Korean coordination falsify Lakoff's claim that extraction out of coordinate structures can violate the ATB constraint as long as the coordinate structure is interpreted sequentially.

In this paper, I will argue that the NTPs in (3) may be either conjuncts or adjuncts. That is, $V$-ko marks either a conjunct phrase in a coordinate structure or an adjunct phrase meaning 'and-then (after)', 'and as a result (cause \& effect)', or 'and nonetheless'. I claim here that the ATB violation is allowed only when an NTP instantiates a head-adjunct structure, but not when it is a coordinate structure. This will enable us to account for the contrast between sentences in (3), where ATB violation is allowed, and sentences in (2), where it is not. I also claim here that the sequential reading of (3) is obtained when the first conjunct is an adjunct, while the non-sequential reading is obtained when the NTPs in (3) are coordinate structures. I will represent $-k o$ used as an adjunct complementizer as $-k o 1$ and the conjunctive $-k o$ as $-k o 2$ in the rest of this paper. Again, the adjunct complementizer $-k o l$ may have at least three subtypes as follows:

(4) The Classification of $-k o$

\begin{tabular}{|c|c|c|c|}
\hline ion & Function & $\underline{\text { Subtype (variants) }}$ & ing \\
\hline 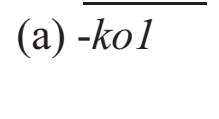 & $\begin{array}{l}\text { Adjunct compl- } \\
\text { ementizer }\end{array}$ & $\begin{array}{ll}\text { i. } & -k o(\text { nase }) \\
\text { ii. } & -k o(\text { se })\end{array}$ & $\begin{array}{l}\text { 'after' or 'and then' } \\
\text { 'cause and effect' }\end{array}$ \\
\hline $\mathrm{ro}_{2}$ & Conjunctive & $\begin{aligned} \text { iii. }- & k o \\
- & -k o\end{aligned}$ & \\
\hline
\end{tabular}

Though it is true that ATB violations are allowed only when the NTP coordination at issue receives a sequential reading, a claim made in this paper is that the distinction between sequential and non-sequential readings in the coordination of NTPs in (3) is a distinction made by syntax, while the sequential vs. non-sequential reading of (2) is derived from semantics or pragmatics. This enables us to maintain the claim that the ATB and the Coordinate Structure Constraint (CSC) hold as syntactic constraints in Korean, since ATB violation is attested only when the NTPs in (3) instantiate a head-adjunct structure.

This paper is organized as follows. In section 1, I provide various linguistic properties of NTPs in coordination to support the claim that the NTPs at issue can be ambiguous between a conjunct and an adjunct analysis, emphasizing that $-k o 1$ and other adjunct suffixes exhibit parallel linguistic behavior. Section 2 shows how to implement the generalizations from the properties of NTPs in coordination 
into HPSG (Sag et al. 2003). On this basis, I will demonstrate how my analysis works for NTPs in coordination. In conclusion, I attempt to show the similarities and differences between Korean NTPs in coordination and English verbal coordination, with respect to extraction.

\section{Properties of Verbal Coordination in Korean \\ 1.1. Phonological Properties of NTPs}

In Korean, the adjunct suffix -kol used in NTPs in coordination, like the adjunct suffix -kose in an after-adjunct, may bear a falling tone or be followed by a pause. In such circumstances, the sentences are read as sequential. In contrast, the conjunct suffix -ko2 'and' does not exhibit such behavior, whether coordination is tensed or non-tensed. This shows that the NTPs interpreted sequentially are similar to adjuncts, which exhibit similar properties.

The sequence $-k o l$ in (5a) can have a falling tone in the Kyung Sang dialect or may be followed by a pause when the reading is sequential. Likewise, there is a falling tone or a pause between the sequence-kose 'after' and the main clause VP as shown in (5b). However, even though the conjunct $-k o 2$ in a coordinate structure with two TPs, as in (6b), may bear a falling tone or be followed by a pause, both the sequential and non-sequential readings are available. (\# stands for a pause or a falling tone.)
a. Kim-i pap-ul mek-ko1 \# kulus-ul chiu-ess-ta.
K-Nom rice-Acc eat-adj.suf dish-Acc clean-Past-Decl
'Kim cleaned the dishes after eating the rice.' (SEQ)
b. Kim-i pap-ul mek-kose \# kulus-ul chiu-ess-ta.
K-Nom rice-Acc eat-adj.suf dish-Acc clean-Past-Decl
'Kim ate the rice and then cleaned the dishes.' (SEQ)
(6) a. Kim-i pap-ul mek-ko2 kulus-ul chiu-ess-ta.
K-Nom rice-Acc eat-Conj dish-Acc clean-Past-Decl
'Kim ate the rice and cleaned the dishes.' (NS)
b. Kim-i pap-ul mek-ess-ko2 kulus-ul chiu-ess-ta.
K-Nom rice-Acc eat-Past-Conj dish-Acc clean-Past-Decl
'Kim ate the rice and cleaned the dishes.' (SEQ or NS)

A coordinate structure with two TPs, as in (7), can have a sequential reading, since the two events, the event of buying the rice and the event of loading it in the car, can be a natural course of events in the world. In this case, we can obtain a sequential reading, regardless of the existence of tone or pause. On the other hand, a sentence with a TP, as shown in (8), can be interpreted either sequentially or non-sequentially, depending on tone or pause. In (8), if a falling tone falls on -ko, the sentence must be interpreted sequentially, whereas if there is no tone the sentence need not be. 
(7)

$\begin{array}{lllll}\text { Kim-i } & \text { ssal-ul } & \text { sa-ess-ko2 } & \text { cha-ey } & \text { sil-ess-ta. } \\ \text { K-Nom } & \text { rice-Acc } & \text { buy-Past-Conj } & \text { car-PostP load-Past-Decl } \\ \text { 'Kim bought the rice and loaded it in the car.' } & \text { (SEQ or NS) }\end{array}$

(8)

Kim-i pap-ul mek-ko(1/2) $\begin{array}{ll}\text { ppang-ul } & \text { mek-ess-ta. } \\ \text { K-Nom rice-Acc eat-Conj } & \text { bread-Acc } \\ \text { eat-Past-Decl }\end{array}$
$\begin{array}{ll}\text { 'Kim ate the bread after eating the rice.' } & \text { (SEQ) } \\ \text { or 'Kim ate the rice and the bread.' } & \text { (NS) }\end{array}$

If a sequential reading is a matter of semantics or pragmatics alone, it is hard to account for why the reading of (8) varies in terms of the existence of tone or pause, but the reading of (6b) and (7) does not. But if NTPs in apparent coordination can also be adjuncts, the phonological property follows.

\subsection{Morphological Properties of NTPs}

Morphologically, the suffix $-k o l$ and other adjunct suffixes such as -kose exhibit similar distributional behaviors in that they both require a non-tensed verbal form in order to be an independent word, as shown in (9).
a. mek-ko1 eat-adj.suf
vs.
*mek-ess-ko1
eat-Past-adj.suf
b. mek-kose
*mek-ess-kose eat-adj.suf eat-Past-adj.suf *mek-ess-koseto
c. mek-koseto vs.
*mek-ess-konase 'cause and effect'
'and (then)' 'nonetheless'
'and then/after'

In addition to that, the verbal form attached to suffix $-k o l$ and adjunct suffixes should be non-stative, as in (10).
a. *alumtap-ko1 vs. *alumtawue-ess-ko1 'and (then)' beautiful(stative)-adj.suf beautiful(stative)-adj.suf
b. *alumtap-kose vs. *alumtawue-ess-kose 'cause and effect'
c. *alumtap-koseto vs. *alumtawue-ess-koseto 'nonetheless'
d. *alumtap-konase vs. *alumtawue-ess-konase 'and then/after'

This similarity in behavior shows us that $-k o l$ can be an adjunct suffix such as 'after'.

\subsection{Syntactic and Semantic Properties of NTPs \\ 1.3.1. Rightward Extraction}

The fact that an element contained in the final verbal phrase of a sentence with a sequentially interpreted NTP can be relativized while an element contained in the final conjunct in TP coordination cannot, regardless of its reading, shows that NTPs are more similar to adjuncts than conjuncts. This is due to the fact that 


\section{Sae-Youn Cho}

extraction is possible from the head daughter in a head-adjunct structure.

The sentence (11) shows that an NP contained in the final VP in a sentence interpreted sequentially can be relativized. Similarly, the NP contained in the final VP in a sentence with an adjunct phrase can also be relativized, as shown in (12). However, the NP contained in the final TP in a TP coordinate structure, as in (13), cannot be moved out.

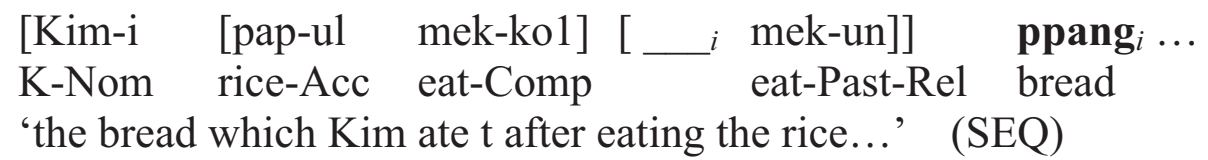

[Kim-i [pap-ul mek-kose] K-Nom rice-Acc eat-Comp(after) 'the bread which Kim ate $t$ after eating the rice...'

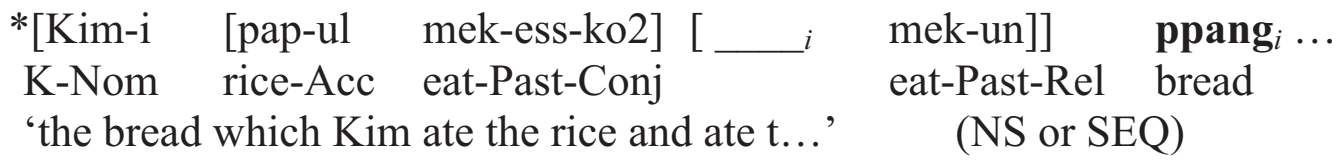

If the first NTP in (11) is an adjunct like (12), the difference in grammaticality between (11) and (13) follows from the CSC, proposed by Ross (1967).

(14) The Coordinate Structure Constraint (CSC)

In a coordinate structure, no conjunct may be moved, nor may any element contained in a conjunct be moved out of that conjunct. (Ross 1967:98-99)

The sentences in (11) and (12) are not subject to the CSC, since we are assuming that the NTP is an adjunct. On the other hand, if sentence (11) is a coordinate structure, we need to explain why (11) is acceptable and (13) is not.

\subsubsection{Leftward Extraction}

An NP contained in the final VP of a sentence containing -kol or a temporal adjunct marker like -kose can be scrambled out of the VP, while an NP contained in the final TP in a TP coordination cannot. Similar to relatives, this also shows that the NTP with $-k o l$ is more like an adjunct phrase than a conjunct. The sentences (15) and (16) licensed by head-adjunct structures are acceptable because they do not violate the CSC, even if the NP is moved out of the main VP, whereas the sentence (17) is unacceptable because the NP is moved from a single conjunct and thus violates the CSC.

$$
\begin{aligned}
& \text { ppang-ul } \left._{i}, \mathrm{Kim}_{\mathrm{i}} \text { [pap-ul mek-ko1] [__ } i \text { mek-ess-ta. }\right] \\
& \text { bread-Acc K-Nom rice-Acc eat-adj.suf eat-Past-Decl } \\
& \text { 'Kim ate the bread after eating the rice.' (SEQ, not NS) }
\end{aligned}
$$



ppang-ul ${ }_{i}, \mathrm{Kim}-\mathrm{i}$
[pap-ul mek-kose] [
mek-ess-ta.]
bread-Acc K-Nom
rice-Acc eat-adj.suf
eat-Past-Decl
'Kim ate the rice and then ate the bread.' (SEQ)
*ppang-ul ${ }_{i}$, Kim-i
[pap-ul
mek-ess-ko2]
bread-Acc K-Nom
rice-Acc
eat-Past-Conj
mek-ess-ta.] eat-Past-Decl
'Kim ate the rice and ate the bread.'
(NS or SEQ)

Semantically, -kol occurs when the NTP containing it delivers sequential meanings such as 'after', 'cause and effect', and 'nonetheless', as illustrated in (18) through (20). The sentences with adjunct suffixes including -kol can undergo preposing, delivering various sequential meanings as shown in the (a) and (b) examples below. In contrast, sentences with $-k o 2$ cannot undergo preposing regardless of the existence of tense inflection or sequential vs. non-sequential reading, as illustrated in the (c) examples below.
a. kulus-ul $_{i}, \quad \mathrm{Kim}-\mathrm{i}$
[pap-ul
mek-ko1] [ dish-Acc K-Nom
rice-Acc
eat-adj.suf
$i$ chiu-ess-ta.]
b. kulus-ul ${ }_{i}$, Kim-i
[pap-ul
mek-konase]
clean-Past-Decl
dish-Acc K-Nom
rice-Acc eat-adj.suf ${ }_{i}$ chiu-ess-ta.]
'Kim ate the rice and then cleaned the dishes.'
c. ${ }^{*}$ kulus-ul $_{i}, \quad$ Kim-i $\quad$ [pap-ul mek-ess-ko2] dish-Acc K-Nom rice-Acc eat-Past-Conj 'Kim ate the rice and cleaned the dishes.' clean-Past-Decl (SEQ 'and then') ${ }_{i}$ chiu-ess-ta.] clean-Past-Decl (SEQ or NS)
a. pay-ka,
Kim-i
[pap-ul
rice-Acc
mek-ko1] ${ }_{i}$ apha-ess-ta.] stomach-Nom K-Nom rice-Acc eat-adj.suf pain-Past-Decl
b. pay-ka, Kim-i [pap-ul mek-kose] [ stomach-Nom K-Nom rice-Acc eat-adj.suf 'Kim ate the rice and as a result had a pain in his stomach.' ${ }_{i}$ apha-ess-ta.] (SEQ 'cause and effect')
c. ${ }^{*}$ pay-ka $i, \quad$ Kim-i [pap-ul mek-ess-ko2] stomach-Nom K-Nom rice-Acc eat-Past-Conj 'Kim ate the rice and had a pain in his stomach.' ${ }_{i}$ apha-ess-ta.] pain-Past-Decl (SEQ or NS)
a. wuncen-ul $l_{i}, \quad$ Kim-i [swul-ul
masi-ko1] [ drive-Acc K-Nom alcohol-Acc drink-adj.suf hay-ess-ta.]
b. wuncen-ul ${ }_{i}$, Kim-i [swul-ul masi-koseto][ drive-Acc K-Nom alcohol-Acc drink-adj.suf ihay-ess-ta.] do-Past-Decl 'Kim drove a car though he drank alcohol a lot.' (SEQ 'nonetheless')
c. *wuncen-ul ${ }^{2}$, Kim-i [swul-ul masi-ess-ko2] [ drive-Acc K-Nom alcohol-Acc drink-Past-Conj do-Past-Decl 'Kim drank alcohol (a lot) and drove a car.' (SEQ or NS) 


\section{Sae-Youn Cho}

\subsection{Generalizations and Their Implications}

From the observations above, we can generalize that like other sequential adjunct suffixes such as -kose, -kol instantiates a head-adjunct structure so that extraction is freely allowed in the sentences with $-k o l$, since the CSC and the ATB are constraints for coordinate structures such as phrases with -ko2. Thus, we can summarize as follows:

(21) The distinction between -ko1 and -ko2 (for Korean)

\begin{tabular}{|c|c|c|}
\hline & $-k o 1$ & $-k o 2$ \\
\hline i. Function & $\overline{\text { (sequential) adjunct suffix }}$ & $\overline{\text { conjunct suffix }}$ \\
\hline ii. Meaning & 'after', 'cause \& effect', 'nonetheless' & 'and' \\
\hline $\begin{array}{l}\text { iii. Instantiating } \\
\text { structure }\end{array}$ & head-adjunct(modifier) & coordinate \\
\hline iv. Stem & non-tensed verb (non-finite) & (finite) verbal \\
\hline v. Constraints & $\mathrm{N} / \mathrm{A}$ & $\mathrm{CSC}$ and ATB \\
\hline
\end{tabular}

Observations from the coordination of NTPs in Korean above give us clues about the similarities and differences between English and Korean verbal coordination. Postal (1998) has provided a syntactic explanation for the three types of 'and' in (22) suggested by Lakoff (1986).

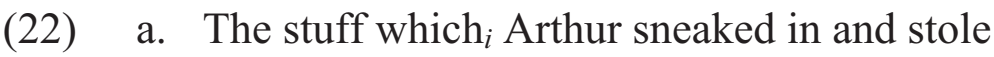
i... (and-then)

b. [How many dogs $]_{i}$, can a person have_ $i$ and still stay sane?

(nonetheless)

c. That is the drug which ${ }_{i}$ athletes take $i$ and become strong.

(cause-effect)

Postal (1998) argues that the phrase of the 'and-then' type in (22a) can be ambiguous between an adjunct and coordinate structure on the basis of syntactic tests such as iterability and deletion. By the same token, phrases like the types 'and-nonetheless' and 'cause-result' in (22b-c) can be analyzed as adjunct structures. This can be summarized as follows:

(23) The distinction adjunct and from conjunction and (for English)

$\begin{array}{lll}\text { i. Function } & \frac{\text { adjunct and }}{\text { (sequential) adjunct comp }} & \begin{array}{l}\text { conjunction and } \\ \text { conjunctive } \\ \text { 'ai. Meaning }\end{array} \\ \begin{array}{lll}\text { iii. Instantiating } \\ \text { structure }\end{array} & \text { head-adjunct(modifier) } & \text { coordinate } \\ \text { iv. Stem } & \text { N/A } & \text { N/A } \\ \text { v. Constraints } & \text { N/A } & \text { CSC and ATB }\end{array}$

The table in (23) showing the characteristics of the English and is quite similar to that in (21) for the Korean - ko. If Postal's (1998) analysis is correct, we 
may argue that the ATB holds as a syntactic constraint in both languages, since ATB violation is attested only when phrases such as NTPs in Korean instantiate a head-adjunct structure.

Before presenting my proposal in detail, I will briefly mention some of the problems found in the Adjunct approach of Yi (1994) and Kim (1995) for the phenomena mentioned above. To capture the syntactic generalizations, the Adjunct approach regards all VP conjuncts except a main VP as adjunct phrases like an after-phrase. ${ }^{1}$ Such an account would, first of all, have to explain why there is no VP coordination in Korean but other coordinations like AP coordination exist. Moreover, though there is no VP coordination, the Adjunct approach still finds it hard to differentiate (1a) from (1b). In other words, it appears that the Adjunct analysis cannot easily account for the fact that extraction out of NTP coordination headed by a non-active verb cannot be allowed but that from NTP coordination headed by an active verb can be allowed. ${ }^{2}$ Furthermore, as illustrated in (2) and (3), it would be a puzzle to the Adjunct approach to show how NTP coordination, in principle, can be interpreted either sequentially or nonsequentially and why sentences undergoing extraction out of NTP coordination must have a sequential reading. However, these difficulties the Adjunct approach faces can be easily avoided under our analysis. ${ }^{3}$

\section{A Constraint-Based Analysis of Coordinate Structures in Korean 2.1. Theoretical Tools}

On the basis of the hypothesis that a set of NTPs may be either adjuncts or conjuncts depending on whether $-k o$ is $-k o l$ or $-k o 2$, I will now present an analysis of these constructions within HPSG (Sag et al. 2003). In HPSG, adjuncts, i.e. modifiers, select their heads so that the MOD value of the adjunct is tokenidentical to the SYNSEM value of its head. Hence, the head-modifier rule in (24) licenses sentences containing NTPs with -kol.

(24) Head-Modifier Rule (Head-Adjunct Rule)

$[$ phrase $] \rightarrow \mathrm{H} 1]\left[\begin{array}{l}\text { COMPS }<> \\ \text { STOP-GAP }<>\end{array}\right]\left[\begin{array}{ll}\text { COMPS } & < \\ \text { MOD } & <1>\end{array}\right]$

Again, we need to specify the information on what the RELATION of the adjunct $-k o l$ can have as its value, since the adjunct suffix -kol may convey one of at least three different sequential readings. To do this, I propose a partial type

\footnotetext{
${ }^{1}$ The difference between the two analyses of the Adjunct approach is that Yi (1994) thinks there to be no VP coordination in Korean while Kim (1995) considers all NTPs to be adjuncts. Though both analyses are different in various respects, I will not pursue that issue here.

${ }^{2}$ The NTP coordination with time adverbs also would be a puzzle to the Adjunct approach. For the relevant data, see Chung (2003).

${ }^{3}$ Chung (2003) also has argued against the so-called Coordination approach of Yoon (1994). Due to space constraints, I will not pursue this here.
} 


\section{Sae-Youn Cho}

hierarchy on predication as in (25) and an exemplar lexical rule for the adjunct suffix -kol whose type is 'and-then' as in (26).

feature structure - predication

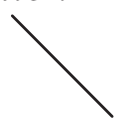

sequentiality

sequential [RELN timely-precede]

and then nonetheless cause and effect ... and

non-sequential

[RELN and then] [RELN nonetheless] [RELN cause and effect]

Suffix -kol Lexical Rule

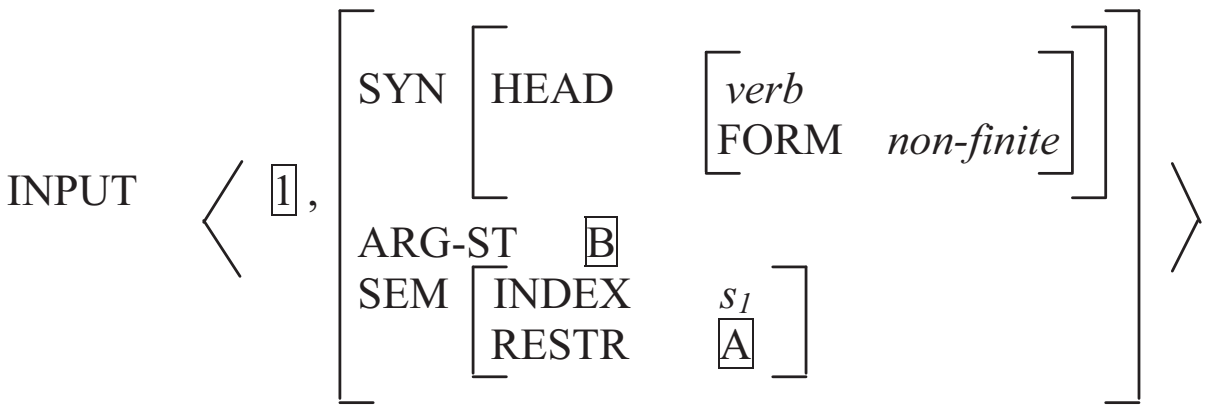

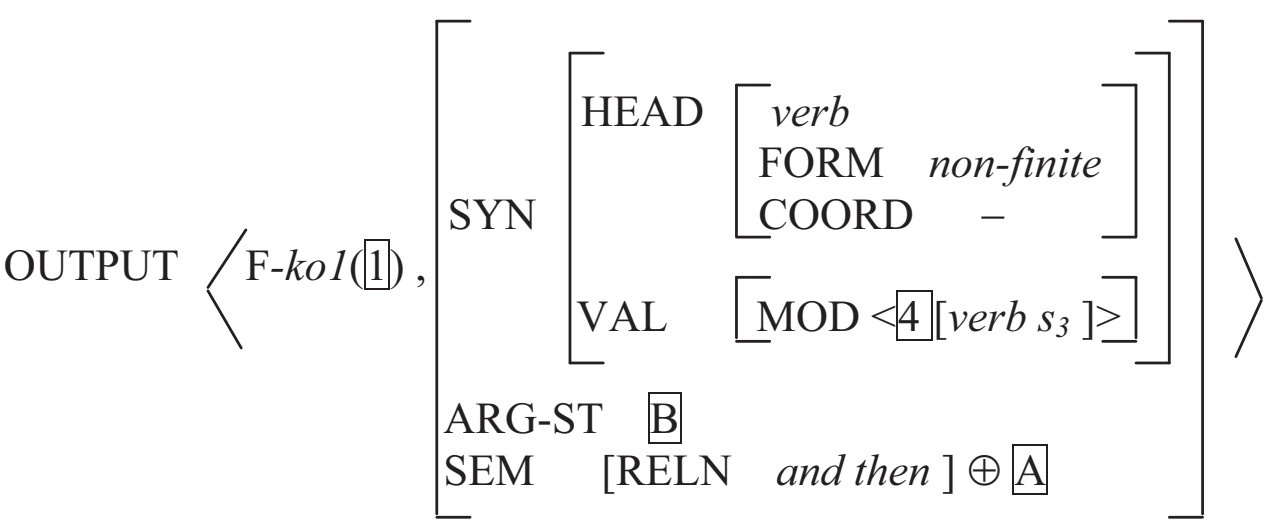

The sequential reading of the sentence with any type of -kol can be obtained in terms of (25), and a more specific reading would be based on which -kol lexical rule is applied among three different $-k o l$ lexical rules.

As for the coordination cases, I postulate two coordination rules for Korean as in (27); one for Symmetric Coordination, where all conjuncts have the same FORM value (for example, [FORM finite]), and the other for Asymmetric Coordination, where all conjuncts do not have the same FORM value. In addition, we need the real conjunct suffix $-k o 2$ as in (28). 
(27) (I) Symmetric Coordination Rule for Korean, Based on Sag et al. (2003)

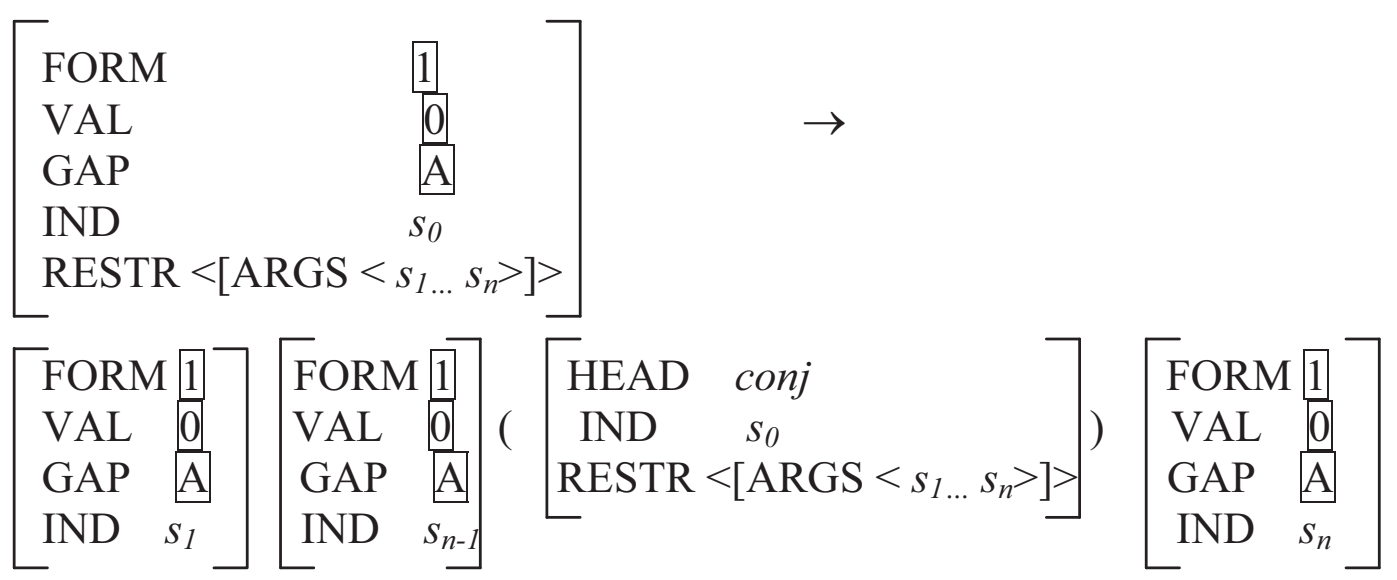

(II) Asymmetric Coordination Rule for Korean, Based on Sag et al. (2003)

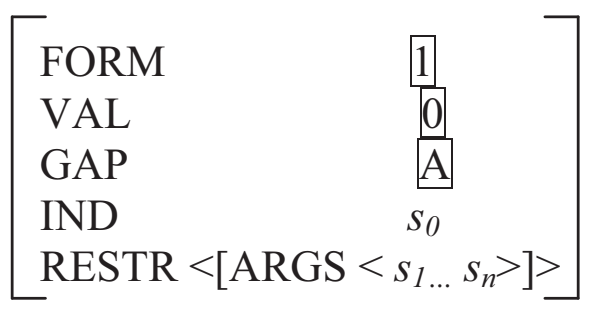

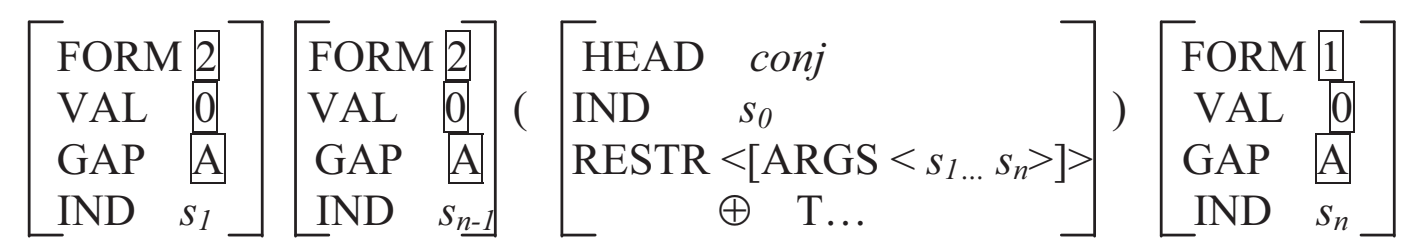

(28) Suffix (Asymmetric) -ko2 Lexical Rule

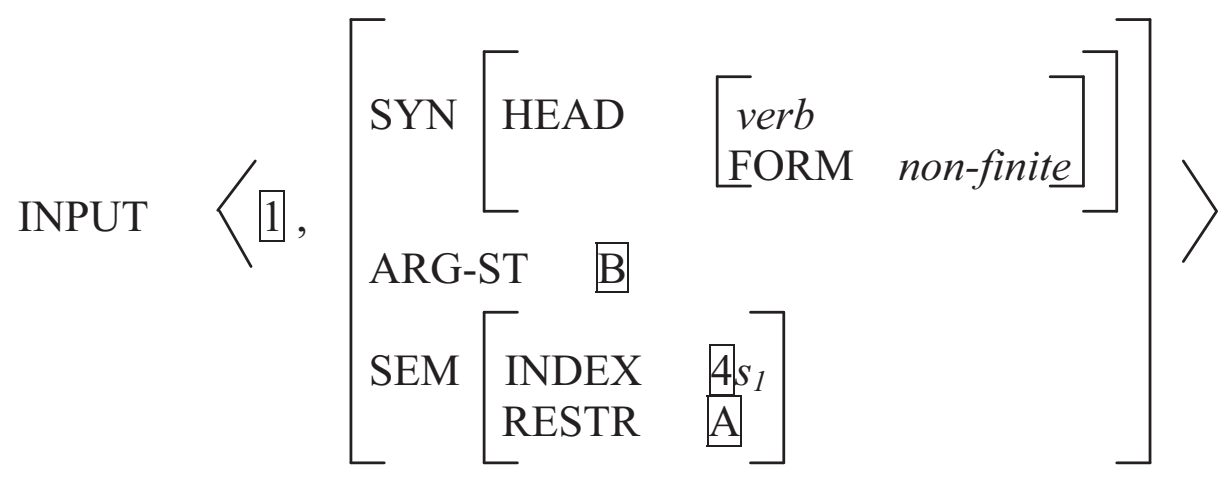




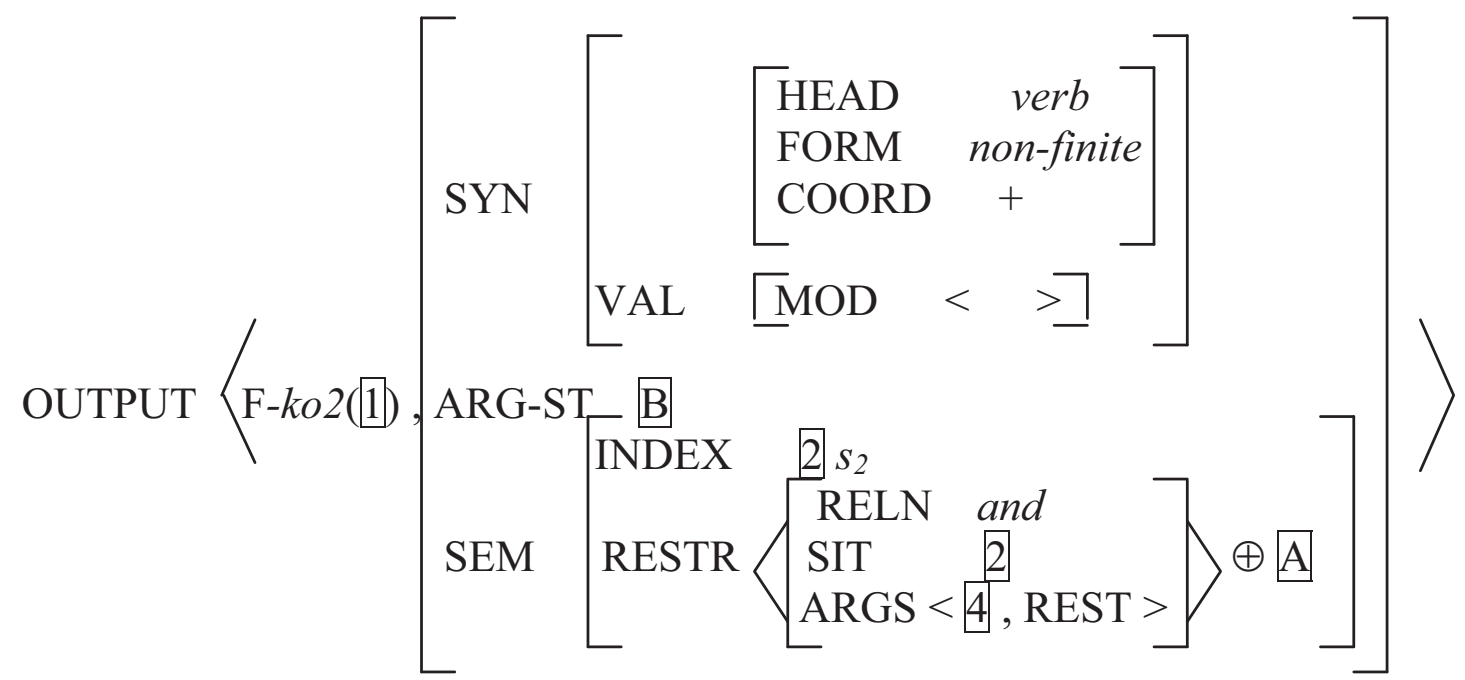

For clarity, I will demonstrate how the theoretical tools work for English Coordination. The coordination rule like (27) enables us to represent a coordinate sentence as in (29):

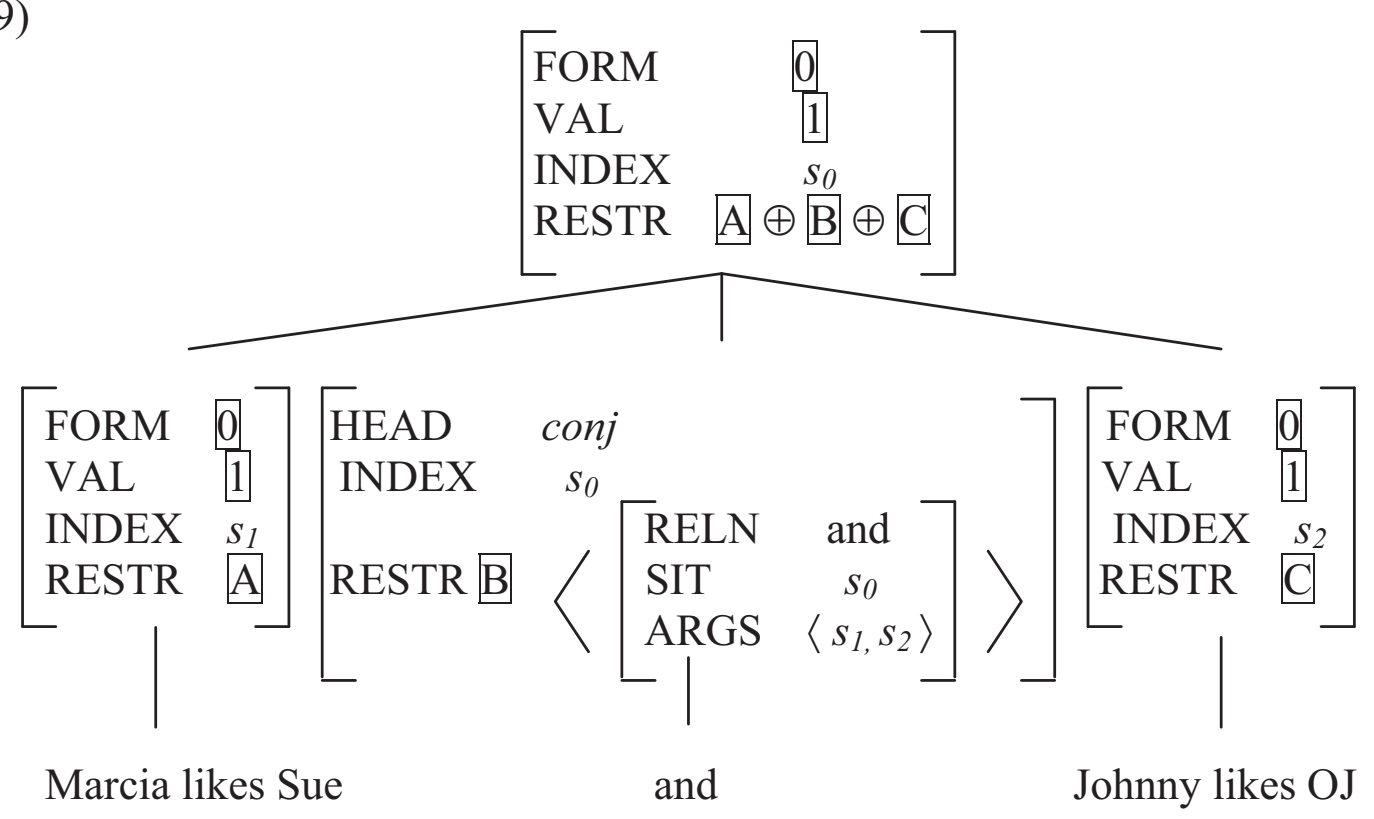

In (29), the FORM value of the first conjunct, finite, is token-identical with that of the second conjunct. In addition, the VAL and GAP values of both conjuncts are the same. The configuration in (29) satisfies all the requirements of the coordination rule in HPSG (Sag et al. 2003), so the sentence, Marcia likes Sue and Johnny likes OJ, is predicted to be grammatical. Sentence (1b), where it violates the CSC, is also correctly predicted to be ungrammatical because the GAP value of the two conjuncts is different, as illustrated in (30). 
(30)

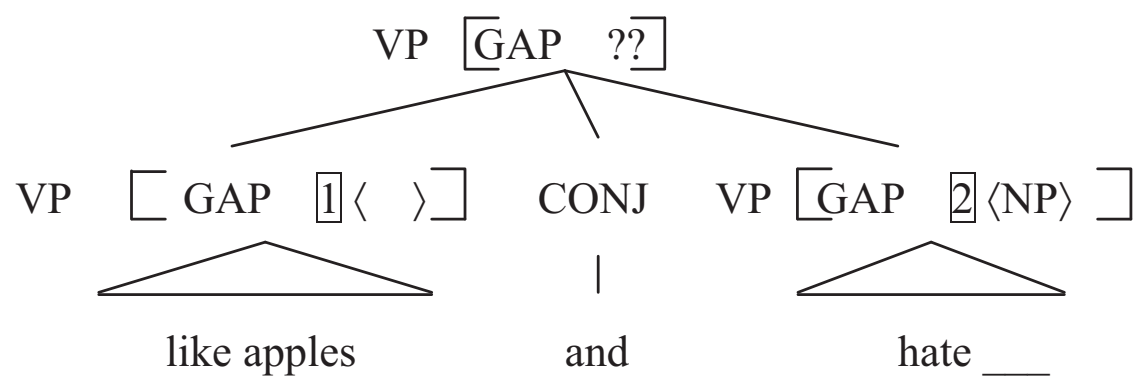

The ATB case in (31), in which all conjuncts have the same GAP value, is predicted to be grammatical by the definition of the coordination rule for English.

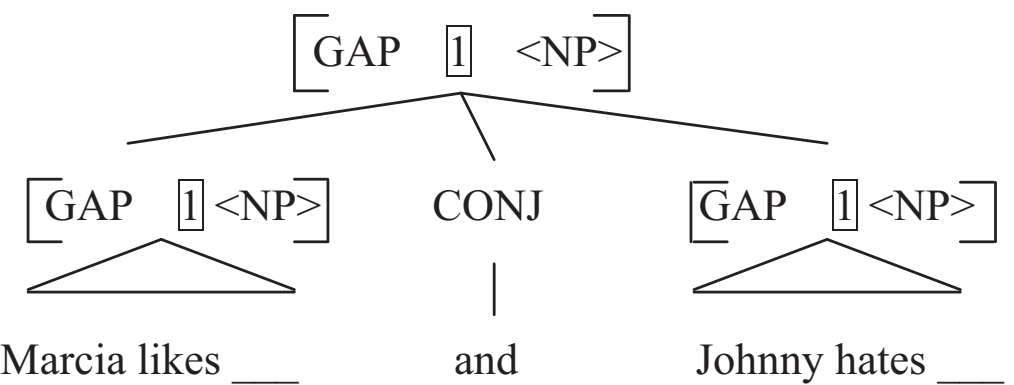

On the basis of the above, I will provide an analysis of Korean verbal coordination in a slightly modified version of HPSG (Sag et al. 2003) in the following section.

\subsection{ATB as a Syntactic Constraint in Korean Verbal Coordination}

\subsubsection{Extraction Out of Real Coordinate Structures}

An NP contained in the final VP conjunct cannot be preposed out of the VP, regardless of whether the sentence receives a sequential or non-sequential reading. The impossibility of the extraction out of real tensed or non-tensed coordination just follows under our analysis, as illustrated in $(32)(=(2 b))$.

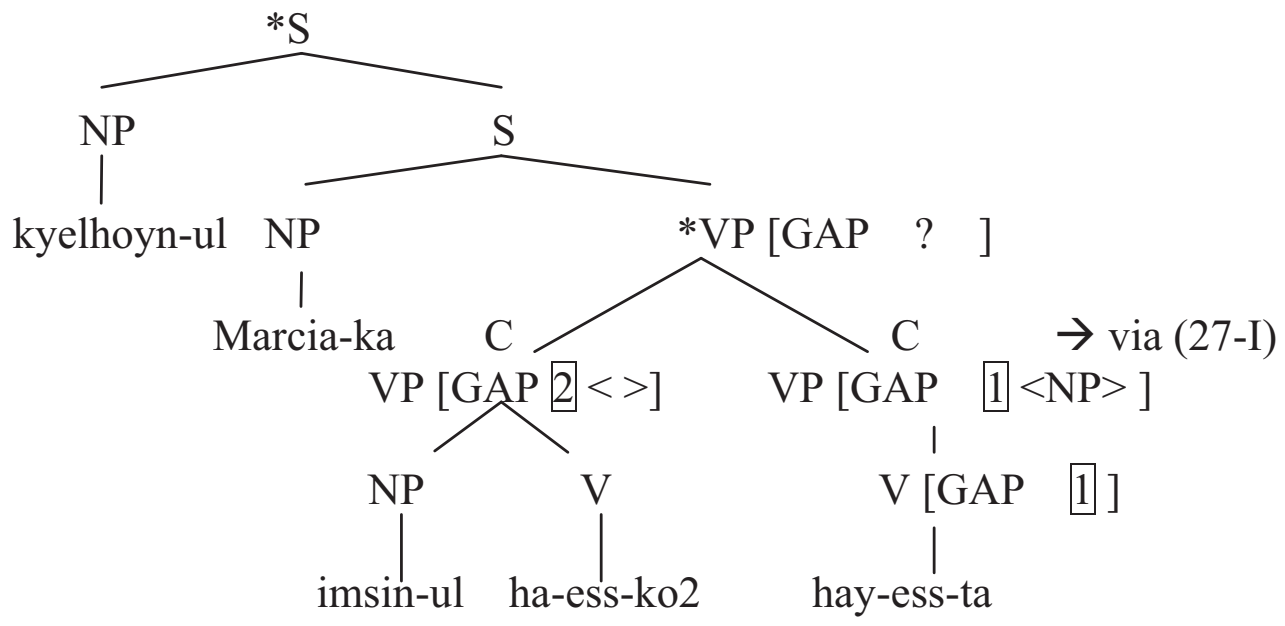




\section{Sae-Youn Cho}

Though sentence (32) can be construed both sequentially and non-sequentially, the information on sequentiality is assumed to be pragmatic, under our analysis. Unlike Lakoff (1986), we claim that the sequentiality delivered from CONTEXT as a RESTRICTION value is not the key factor on whether or not extraction is possible in coordinate structures. Hence, extraction out of the coordinate structures headed by $-k o 2$ cannot be allowed, as shown in (32). Specifically, the NP, kyelhoyn-ul, cannot be extracted from the second conjunct in (32) because both conjuncts must have the same GAP value by the definition of the symmetric coordination rule for Korean in (27-I). As for sequentiality, sentence (32) may have a sequential reading, depending on the CONTEXT value, which does not affect the possibility of the extraction at issue.

\subsubsection{Extraction out of Head-Adjunct Structures}

An NP contained in the main VP in a sentence with NTPs can be preposed out of the VP only when the sentence receives a sequential reading. Further, we have claimed that the extraction is attested only when the phrases with NTPs at issue instantiate head-adjunct structures conveying some predication as a subtype of sequential in (25). Given (24)-(26), we can represent sentence (3b) as in (33).

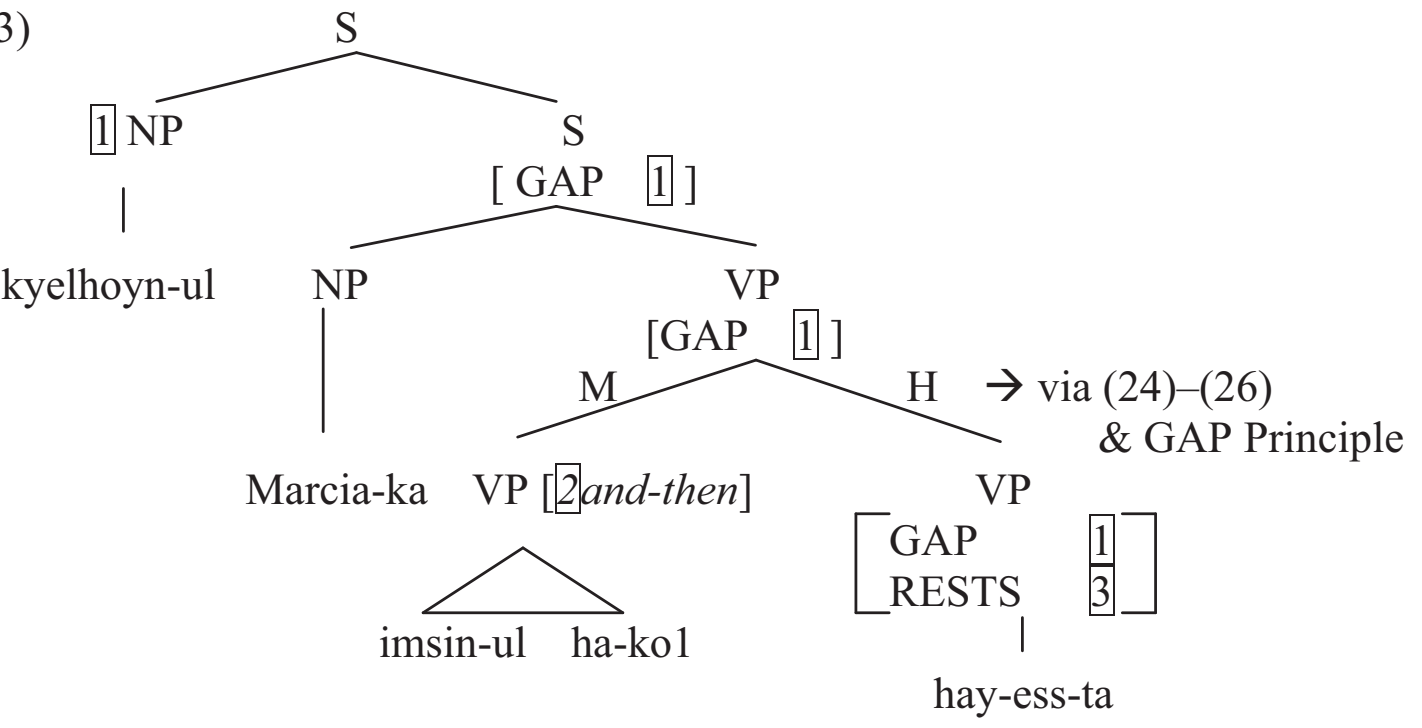

Sentence (33), where an NP is extracted from the final VP, is predicted to be grammatical under our analysis, since it satisfies the requirements of (24)-(26). Specifically, the NP extracted out of the VP in (33) is permissible since the two VPs instantiate a head-adjunct structure so that it need not obey the CSC. Hence, extraction in (33) is legal.

\section{Conclusion}

On the basis of the hypothesis that NTPs in coordination may be either adjuncts or conjuncts, I have argued that the (im)possibility of the extraction from Korean verbal coordination can be predicted by syntactic structures, rather than by appeal 
to semantics or pragmatics. This explains various factors about the prosodic, morphological, and syntactic and semantic characteristics of non-tensed phrases in Korean verbal coordination. The fact that syntax is responsible for these characteristics is further supported by the fact that even though both the sequential and non-sequential readings are available in TP coordination, they do not allow extraction in violation of CSC or ATB. This shows that Korean coordination cannot be accounted for in the manner suggested by Lakoff (1986) for English coordination. Hence, Korean coordination observes the ATB and CSC constraints as syntactic constraints. Furthermore, Postal (1998) has claimed that the three types of and suggested by Lakoff (1986) can be syntactically defined, which is summarized in (23). If Postal's (1998) analysis is on the right track, we could claim that the verbal coordination in both languages observes the ATB and CSC constraints as syntactic constraints.

\section{References}

Chung, Daeho. 2003. On conjunct size in Korean -ko coordination, Japanese/Korean Linguistics 11:471-484.

Kim, Jong-Bok. 1995. The grammar of negation: A lexicalist, constraint-based perspective. Ph.D. diss., Stanford University.

Lakoff, George. 1986. Frame semantic control of the coordinate structure constraint. $C L S$ 22. Chicago: Chicago Linguistic Society.

Postal, Paul M. 1998. Three Investigations of Extraction. Cambridge, MA: MIT Press.

Ross, John. 1967. Constraints on variables in syntax. Ph.D diss., MIT. Distributed by the Indiana University Linguistics Club.

Sag, Ivan A., T. Wasow, and Emily M. Bender. 2003. Syntactic Theory: A Formal Introduction. $2^{\text {nd }}$ Edition. Stanford, CA: CSLI.

Yi, Eun-Young. 1994. Adjunction, coordination, and their theoretical consequences. Paper presented at the International Circle of Korean Linguistics, University of London, July 1994.

Yoon, James H.-S. 1994. Korean verbal inflection and checking theory. MIT Working Papers in Linguistics, Vol. 22: Morphology-Syntax Connection, 251-270. Cambridge, MA: Department of Linguistics and Philosophy, MIT.

Department of English Language and Literature

Honam University

59-1, Seobong-dong, Gwangsan-gu,

Gwangju, Korea

506-714

sycho@honam.ac.kr 